\title{
Integrated Analysis of Permeability Reduction Caused by Polymer Retention for Better Understanding Polymer Transport
}

\author{
ByungIn Choi, ${ }^{1}$ Jinsuk Choi, ${ }^{2}$ and Kun Sang Lee ${ }^{2}$ \\ ${ }^{1}$ Computer Modelling Group Ltd., Building No. 12, Office 320, Dubai International City, P.O. Box 500 446, Dubai, UAE \\ ${ }^{2}$ Department of Natural Resources and Environmental Engineering, Hanyang University, 222 Wangsimni-ro, Seongdong-gu, \\ Seoul 133-791, Republic of Korea \\ Correspondence should be addressed to Kun Sang Lee; kunslee@hanyang.ac.kr
}

Received 27 November 2014; Accepted 2 June 2015

Academic Editor: Rafael García-Tenorio

Copyright ( $\odot 2015$ ByungIn Choi et al. This is an open access article distributed under the Creative Commons Attribution License, which permits unrestricted use, distribution, and reproduction in any medium, provided the original work is properly cited.

\begin{abstract}
Polymer retention is one of the most important factors to govern polymer propagation through porous media, determining whether successful or not. The focus of previous studies has been limited to polymer concentration loss caused by the retention; not only change in polymer concentration, but also reduction in reservoir permeability is the main issue for theoretical transport study. Due to the lack of accuracy of Langmuir isotherm describing the polymer retention mechanisms, this study proposes a new type of matching interpretation method to correlate the permeability reduction factors from experiments to permeability. In order to solve the problem of poorly matching results between estimation and observation, use of nonadsorptive constant conditionally selected in matching process was made. Based on the threshold permeability reduction factors, approximate critical permeability can be calculated to which nonadsorptive constant would be applied. Results showed significant improvements in the estimation of permeability reduction for both low and high permeability cores. In addition, effects of permeability reduction on polymer transport in field scale were analyzed using the proposed matching model. Thus, not only does this interpretation method help to evaluate prediction for accurate flow behavior, but also unwanted risk can be evaluated.
\end{abstract}

\section{Introduction}

Use of a polymer flood is a technique to enhance oil recovery (EOR) by improving reservoir sweep and reducing the amount of injection fluid needed to recover a given amount of oil. A number of papers on the application and benefits of polymer flood have been published [1-4]. Adequate polymer propagation in the porous media is one of the most important requirements to achieve a successful field application. Polymer retention can heavily impact propagation. Polymer particles always interact with the rock formations during transport through the reservoir. The adsorption is one of the whole kinds of retention mechanisms; however, sometimes total retention is represented as only adsorption.

Reservoir properties are the primary factors governing fluid transport and reservoir performance [5-7]. For these reasons, the polymer retention has been studied, associated with a lot of reservoir properties such as permeability, pore shape, saturation, and contents of rocks. However, the verification or interpretation of retention process has been limited in terms of polymer concentration loss not only in lab tests, but also in reservoir simulations. Even though effect of polymer retention, commonly referred to as permeability reduction, is also an essential parameter to estimate the polymer transport through the reservoir, deficiency in the quantities as well as qualities was observed. Some reasons why relatively little endeavor was put into the focus on permeability reduction are as follows. The first key reason is the absence of accurate model to describe the acquired measurement because permeability reduction is a result caused by multiplication of complicated mechanisms, not only by one mechanism. It shows a poor estimation where matching results were interpreted based on the Langmuir isotherm model with assuming that the permeability reduction is caused only by polymer adsorption. Ironically, most chemical EOR reservoir simulators use the Langmuir isotherm as a fundamental model to reflect permeability reduction factors. Another reason is that there was no significant need 
for interpretation between permeability and permeability reduction factors even though permeability reduction is strongly related to the permeability. The reduction factor increases dramatically with decreasing permeability. Considerable scattering was also observed in the correlation of permeability reduction to low permeability. Until recently, most polymer EOR cases were applied in very permeable formations over around $e^{-12} \mathrm{~m}^{2}[8]$. The estimation value according to low permeability below $0.5 e^{-12} \mathrm{~m}^{2}$ is not widely used or applicable. However, needs for extended application of polymer flooding into low permeability formations due to the shortage of permeable targets are requiring more accurate estimation even in the low permeability ranges. Final reasons are caused by the intrinsic nature of permeability reduction as a relatively ambiguous parameter including too many associated terms such as changes in viscosity, depleted pore size, adsorbed particle, and pore shape. As a result, it is not possible to establish a reasonable mathematical model considering the whole parameters mentioned above. In addition, the interpretation of the experimental results of polymer retention correlating with permeability is too complicated to apply to real reservoir conditions. Typically, experimental results have been obtained and interpreted in terms of the main mechanism of polymer retention: polymer adsorption [9-12]. After that, it is necessary to extend the estimation for reservoir conditions based on experimental results because conducting experiments at the whole range of reservoir permeabilities is costly and unrealistic. However, previous laboratory studies on the relationship between retention and permeability yielded an inaccurate and limited interpretation. The inaccuracy shown in the analyses was due to the nonuniform contribution of polymer adsorption, depending on permeability. The obtained permeability reduction factor, defined as a numerical term of residual resistance factor (RRF), results from a depleted pore radius and decrease in the number of flow channels by polymer retention consisting of two main mechanisms: mechanical entrapment and adsorption. Even though these two mechanisms are definitely distinct processes, their interpretation for the permeability reduction factor has been limited to the effect of adsorptive polymer only. To overcome limitations in the previous technical interpretation of experimental results, a new correlation between permeability reduction and variation in permeability was developed. This semianalytical approach provided a better matching estimation to acquire permeability-dependent RRF. This work could be a helpful guideline for predicting uncertainty from the polymer retention prior to field implementation in the heterogeneous fields.

For the successful application of polymer enhanced oil recovery (EOR) projects, the polymer retention must be evaluated prior to field implementation because the ability to predict the influence of retention on the transport of polymer solution is vital to the proper design of field scale projects.

\section{Background Theories}

2.1. Polymer Transport. Mass transport and fluid flow in porous media are widely utilized in the fields of geoscience and petroleum engineering. Both polymer transport and fluid flow through porous medium are characterized based on the Darcy flow, relative permeability, and particle transport [13]. The basic formula used to describe fluid flow in porous medium is as follows:

$$
\frac{\partial}{\partial t}\left(\phi \widetilde{C}_{\kappa} \rho_{\kappa}\right)+\nabla \cdot\left[\sum_{l=1}^{n_{p}} \rho_{\kappa}\left(C_{\kappa l} \mathbf{u}_{l}-\mathbf{D}_{k l}\right)\right]=0
$$

where $\kappa$ is the component index, $l$ the phase index, $\phi$ the porosity, $\widetilde{C}_{\kappa}$ the overall concentration, $\rho_{\kappa}$ the density of component, $\mathbf{u}_{l}$ the Darcy velocity of phase $l$, and $\mathbf{D}_{\kappa l}$ the dispersion tensor.

Among the three governing concepts, a relatively small contribution is drawn from particle transport to the numerical simulations, although this term plays an important role in polymer transport. Conventional numerical approaches are limited to the Langmuir isotherm, which is a very simple and strong function used in homogeneous cases. However, there were critical reports on the complexity of adsorption and poor estimation achieved when applying it to heterogeneous porous media due to the complex retention phenomena [14-18]. This paper examines the effects of heterogeneous characteristics of porous media on polymer transport.

2.2. Heterogeneity in Permeability versus Polymer Adsorption. Polymer flooding applied in heterogeneous reservoirs causes an unexpected problem due to its high level of adsorption. In moderately heterogeneous fields, polymer flooding can show effective performance while strongly retained polymer particles induce restricted flow channels and poor penetration. The high contrast of permeabilities prevents a polymer solution from penetrating into the low permeability layers. Thus, the strongly reduced flow in low permeability leads to a deeper penetration only into higher permeability zones [16].

Polymer adsorption properties such as retention of macromolecules, residual resistance factor, inaccessible pore volume, and desorption level depend on the formation permeability. Heterogeneities in petrophysical properties, especially in permeability, can result in these adsorption properties varying significantly within a reservoir system. For example, the adsorbed polymer induces greater resistance to flow in the low permeability layers than in the high permeability layer $[14,19,20]$. According to the Jewett and Schurz experimental works [9], they found that no accurate correlations between residual resistance factors and permeability were available. The intricate correlation between adsorption and variation in permeability prevents operators from predicting polymer transport under such reservoir conditions. However, the phenomenon should be accurately analyzed because the effectiveness of polymer flooding decreases significantly with increasing adsorption. Szabo [21] also reported on the significance of adsorbed polymers depending on the permeability distribution, showing that polymer adsorption is more likely to take place in a low permeability formation. The adsorbed polymer leads to irregular propagation associated with complex flow behavior and poor performance in the heterogeneous fields. Therefore, 
a polymer adsorption test in heterogeneous reservoir conditions should be accurately analyzed prior to application of polymer flooding in reservoir scale.

\section{Mathematical Backgrounds}

Analysis of general adsorption has been conducted with a conventional Langmuir-type isotherm as a function of concentration and temperature. The Langmuir-type isotherm is given by the formula

$$
\widehat{C}_{p}=\min \left[C_{p}, \frac{a_{p}\left(C_{p}-\widehat{C}_{p}\right)}{1+b_{p}\left(C_{p}-\widehat{C}_{p}\right)}\right],
$$

where $C_{p}$ is the injected polymer concentration (gmole $/ \mathrm{m}^{3}$ ), or, in general, the polymer concentration before adsorption, $C_{p}-\widehat{C}_{p}$ is the equilibrium concentration in the rock-polymer solution system (gmole $/ \mathrm{m}^{3}$ ), and $a_{p}$ and $b_{p}$ are the empirical constants.

Given the general Langmuir isotherm, Langmuir's coefficient was detailed to figure out the variation of adsorption depending on permeability. The constant $a_{p}$ is defined as

$$
a_{p}=\left(a_{p 1}+a_{p 2} C_{\mathrm{sep}}\right)\left(\frac{k_{\mathrm{ref}}}{k}\right)^{0.5},
$$

where $a_{p 1}$ and $a_{p 2}$ are input or fitting parameters and $k_{\text {ref }}$ is the reference permeability $\left(\mathrm{m}^{2}\right)$ of the rock used in the laboratory measurement. Based on Langmuir's coefficients, the adsorption level can be calculated as a function of permeability. After the adsorption, the adsorbed polymer is attributed to the restriction of flow of macromolecular polymers. The adsorption mechanism occurs in all pore walls where the polymer particles can get access. Flow resistance to the injected polymer solution takes place as a result of polymer adsorption. The reduction in permeability is postulated to be due to an adsorbed layer of polymer molecular coils that reduces the effective size of the pores. A dimensionless number has been formulated to correlate the permeability reduction factor, defined as the RRF, with the rock properties. This dimensionless number represents the ratio of the size of the polymer molecular coil to an effective pore radius of the porous medium [22].

The RRF is defined as

$$
\mathrm{RRF}=\frac{\Delta p_{W F} \text { before polymer flooding }}{\Delta p_{W F} \text { after polymer flooding }}
$$

and this equation implies that permeability reduction is caused by polymer flooding. In the numerical approach, permeability reduction factor $\left(F_{k r}\right)$ is normally expressed as follows:

$$
F_{k r}=1+\left(F_{k r, \text { max }}-1\right) \frac{\widehat{C}_{p}}{\mathrm{AdC}},
$$

where $F_{k r \text {, max }}$, dimensionless, is the maximum permeability reduction factor, AdC is the maximum adsorption capacity $\left(\mathrm{gmole} / \mathrm{m}^{3}\right)$, and $\widehat{C}_{p}$ is the residual adsorption level $\left(\right.$ gmole $\left./ \mathrm{m}^{3}\right)$.
TABLE 1: Experimental data for polymer adsorption.

\begin{tabular}{lc}
\hline $\begin{array}{l}\text { Permeability, } \\
k\left(e^{-12} \mathrm{~m}^{2}\right)\end{array}$ & $\begin{array}{c}\text { Residual resistance factor, RRF } \\
\text { (dimensionless) }\end{array}$ \\
\hline 2.000 & 1.9 \\
0.980 & 2.3 \\
0.450 & 3.0 \\
0.254 & 3.9 \\
0.142 & 5.2 \\
0.069 & 7.3 \\
\hline
\end{tabular}

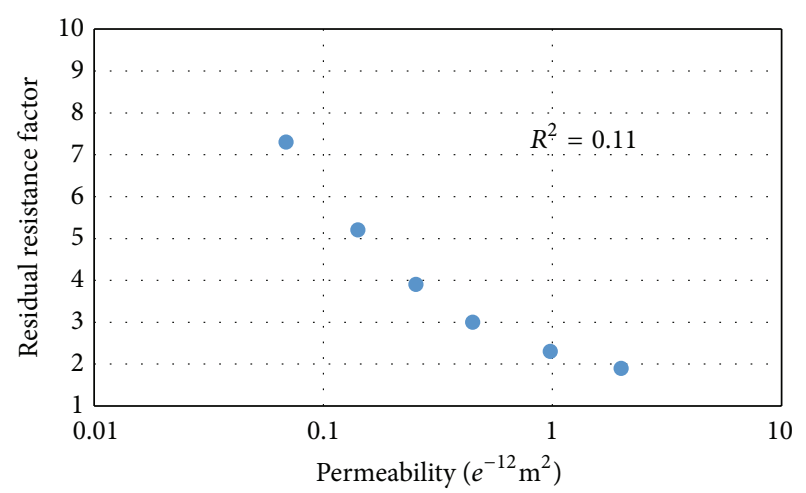

FIGURE 1: Residual resistance factors versus rock permeability, caused by polymer adsorption [10].

There is little experimental data on permeability reduction factor caused by polymer injection $[10,23]$. Table 1 shows the permeability reduction factors at various permeabilities acquired from experiments conducted by Jennings et al. [10]. According to Figure 1, the permeability reduction factors vary inversely with permeability. They reported the dependence of the resistance factor produced by adsorption on the pore size as a reversely proportional relationship. In other words, the resistance factor increases as permeability decreases. Various permeability reduction factors, as one sample of RRF $=5$ for rocks with $0.15 e^{-12} \mathrm{~m}^{2}$, were observed ranging from 1 to 10 , depending on the permeability.

\section{Interpretation of Permeability versus RRF}

4.1. Matching Model. In order to interpret the dependency of adsorption on heterogeneous permeability, a mathematical concept from average pore diameter based on the capillary bundle model of a porous medium, the Hagen-Poiseuille equation was used:

$$
\bar{v}=\frac{R^{2} \Delta p}{8 \mu(\Delta L)},
$$

where $\bar{v}$ is the average interstitial pore velocity ( $\mathrm{m} / \mathrm{sec}), \mu$ the viscosity (poise), $\Delta L$ the length of pipe $(\mathrm{m}), R$ the capillary radius $(\mathrm{m})$, and $\Delta p$ the pressure loss $(\mathrm{kPa})$. By comparing with the Darcy equation,

$$
\bar{v}=\frac{k \Delta p}{\phi \mu(\Delta L)},
$$


pore radius was acquired in the form

$$
r=\left(\frac{8 k}{\phi}\right)^{1 / 2} .
$$

Based on the above equation, permeability reduction by polymer adsorption can be calculated as being reversely proportional to permeability. For instance, if the permeability increases 10 times, permeability reduction can be decreased by a factor of the square root of 10 . In addition, analytical measurement for permeability reduction can be accounted for based on the depleted layer model, as shown in [24]

$$
\mathrm{RRF}=\left(1-\frac{e}{r}\right)^{-4},
$$

where $r$ is the pore radius and $e$ is the thickness of the adsorbed layer.

This model is able to describe a quantitative change in pore size after polymer attachment to the rock surface. Thus, a basic approach to find a correlation function commences at the general form of a second-order rational function, defined as

$$
\mathrm{RRF}=\frac{a}{k^{2}}+b
$$

A physical boundary concept should be applied for acquiring a constant $b$ :

$$
\lim _{k \rightarrow \infty} \operatorname{RRF}(k)=1
$$

for constant $b$ to reach 1 in the above equation. $\mathrm{RRF}=1$ means that there are no permeability reductions due to a significantly larger pore diameter compared to a very small reduction in size caused from attached polymer particles.

To build up the mechanic correlation model between permeability and RRF, fitting constant $a$ was calculated by matching the general form of $\mathrm{RRF}=a / k^{2}+1$ with observational results. However, another interesting result was observed, as shown in Figure 2. Results indicated that the trend line accounting for the correlation between permeability and adsorption has inaccurate matching depending on the range of permeability. Poor matching with a poorly correlated $R$ squared coefficient and scattering were observed in the low permeability range compared to relatively favorable results achieved over the intermediate range of permeability.

Unusual trend shown in the mechanistic matching can be explained by two possible reasons. The first reason is attributed to mechanical trapping in a low permeability porous medium. This mechanical trapping is generally higher than in a high permeability medium, leading to significant increases in the residual resistance factor. The mechanism, however, cannot be taken into account in the interpretation by the adsorptive process. Mechanical trapping is more likely a mechanism associated with a reduction in the number of channels, not with a reduction of flow cross-section. Another reason can be derived from the physical assumption for employing Langmuir's adsorption interpretation, particularly in single layer attachment. For rock with high clay content or

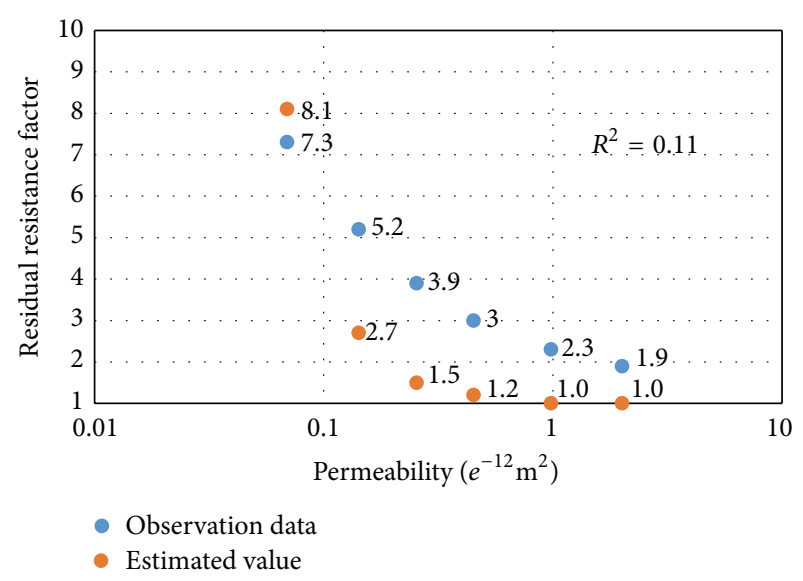

FIGURE 2: Matching results between observation and estimation using theoretical correlation function of $\mathrm{RRF}=a / k^{2}+1$.

exposure to high fluid velocity, polymer particles can attach in layers that are double or more [18]. Thus, the higher permeability reduction intertwined with multilayered adsorption cannot be explained by the conventional adsorption model in which permeability reduction is based on a depleted layer concept assuming single attachment of polymer molecules onto rock surfaces.

To analyze permeability reduction factors from a higher level of adsorption in low permeability rocks more accurately, we tried to implement new matching functions depending on rock permeability. $R$ squared coefficients were also used to verify their accordance in the matching process. As explained above, the large size of the EOR polymer can result in mechanical trapping, which occurs more frequently in a low permeable porous medium than in a high permeable. This large contribution to permeability reduction is one of the mechanical trapping's outstanding characteristics. Considering the mechanical trapping phenomenon, the constant $b$ was modified in the matching function, which accounts for the additional permeability reduction caused from the nonadsorptive mechanism. The matching function is proposed as follows:

$$
\mathrm{RRF}=\frac{a}{k^{2}}+C_{\mathrm{M} . \mathrm{Trap}}+1,
$$

where $C_{\text {M.Trap }}(>0)$ is the fitting constant for the nonadsorptive mechanism. Although (12) has a form similar to that of (10), the mechanical trapping constant, $C_{\mathrm{M} \text {.Trap }}$, cannot be 1 in this matching function. The following results show not only the verification of (12), but also its limitation when applied in a high permeability region. Figure 3 indicated that the matching output could be significantly improved in the region below permeability of $0.45 e^{-12} \mathrm{~m}^{2}$ with a new correlation. As shown in Table 2 , a high permeability range over $0.45 e^{-12} \mathrm{~m}^{2}$ yields worse performance compared with that from (10). The conflicting output for different permeability regions comes from the presence of a nonadsorptive constant, $C_{\text {M.Trap }}$. The coefficient represents the extra permeability reduction caused by other mechanisms except adsorption. Among these mechanisms, mechanical trapping contributes significantly 


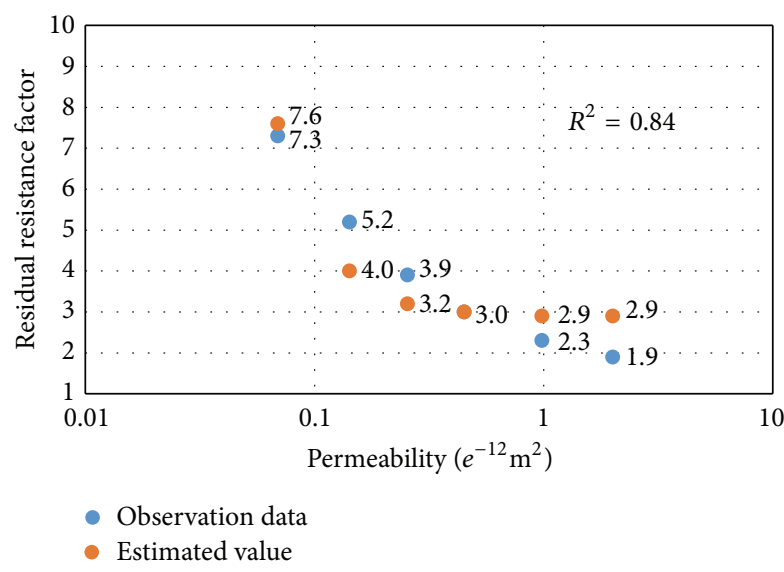

FIGURE 3: Matching results between observation and estimation using theoretical correlation function of $\mathrm{RRF}=a / k^{2}+C_{\mathrm{M} \text {. Trap }}+1$.

TABLE 2: Observation and estimation of RRF at various permeabilities.

\begin{tabular}{lcc}
\hline $\begin{array}{l}\text { Permeability, } \\
k\left(e^{-12} \mathrm{~m}^{2}\right)\end{array}$ & $\begin{array}{c}\text { Residual resistance factor, RRF } \\
\text { (dimensionless) }\end{array}$ & $\begin{array}{c}\text { Estimated } \\
\text { values }\end{array}$ \\
\hline 2.000 & 1.9 & 2.9 \\
0.980 & 2.3 & 2.9 \\
$\mathbf{0 . 4 5 0}$ & 3.0 & 3.0 \\
0.254 & 3.9 & 3.2 \\
0.142 & 5.2 & 4.0 \\
0.069 & 7.3 & 7.6 \\
\hline
\end{tabular}

to permeability reduction by blocking pores of relatively small diameter. However, this pore-blocking mechanism can take place only when flow channels with small diameters appear in the porous media. For a low permeability core, it is more likely to have a large number of pore throats with relatively small diameters, while there must be a small number of pores in the high permeability cores. Thus, the additional reduction produced from mechanical trapping, $C_{\text {M.Trap }}$, should be limited to the cases with low permeability.

Determining a critical permeability for application of the mechanical trapping constant was conducted based on whether an estimation was shifted closely into the observation value or not. In addition, overestimation for high permeability ranges was regarded as a nonphysical result. An increase in RRF estimation for permeability over $0.45 e^{-12} \mathrm{~m}^{2}$ was considered to be not a reasonable value. In this study, the critical permeability for application of $C_{\text {M.Trap }}$, therefore, was calculated as $0.45 e^{-12} \mathrm{~m}^{2}$, which is a critical point that is very consistent with other experiments reporting on permeability range $\left(\approx 0.5 e^{-12} \mathrm{~m}^{2}\right)$ where RRF was significantly reduced $[8$, 24]. Results from cores with permeabilities over $0.45 e^{-12} \mathrm{~m}^{2}$ can be interpreted by employing matching type 2 expressed in (13). In contrast, general type 1 can be used to estimate results in cores with permeabilities less than $0.45 e^{-12} \mathrm{~m}^{2}$. A better fit than previous matching function correlation could

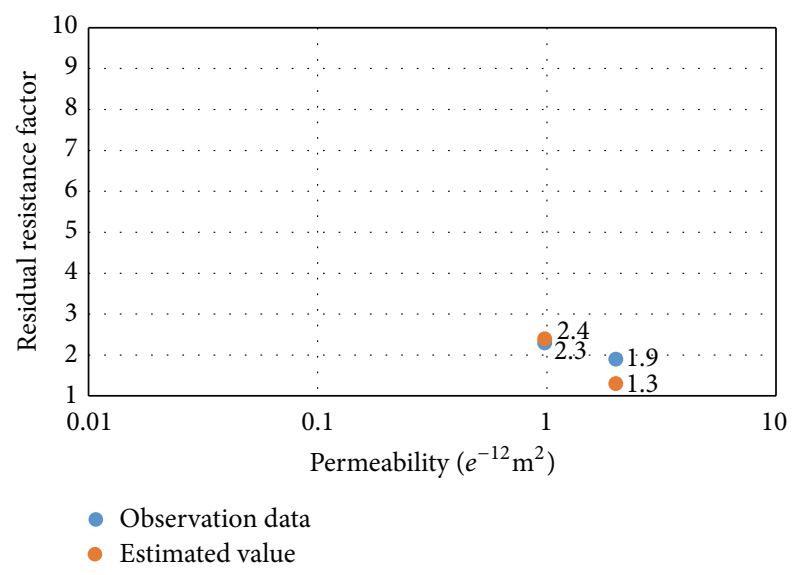

FIGURE 4: Matching results between observation and estimation using the correlation function of RRF $=a_{2} / k^{2}+1$.

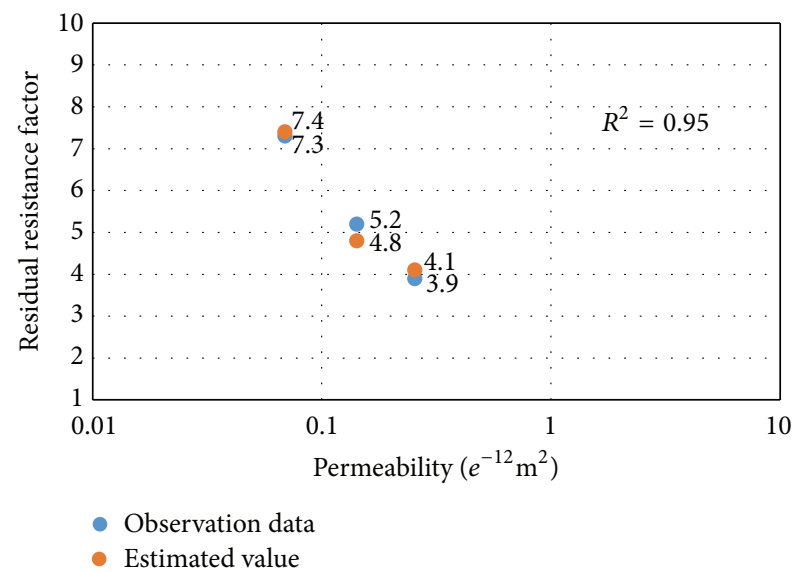

FIGURE 5: Matching results between observation and estimation using the correlation function of $\mathrm{RRF}=a_{1} / k^{2}+C_{\mathrm{M} \text {.Trap }}+1$.

be obtained through the use of selective fitting constants according to permeability range as follows:

$$
\begin{aligned}
& \mathrm{RRF}=\frac{a_{1}}{k^{2}}+C_{\mathrm{M} . \text { Trap }}+1 \quad \text { for } k \leq 0.45 e^{-12} \mathrm{~m}^{2}, \\
& \mathrm{RRF}=\frac{a_{2}}{k^{2}}+1 \quad \text { for } k>0.45 e^{-12} \mathrm{~m}^{2} .
\end{aligned}
$$

Figures 4 and 5 illustrate separate applications where the correlation function yields a significant improvement in estimation for the cases in both low and high permeability ranges. Estimated values in high permeability conditions show tolerant differences from observations, as shown in Table 3 . The results in low permeability rock also verify the good estimation in the cases from 0.07 to $0.25 e^{-12} \mathrm{~m}^{2}$, yielding a very highly correlated coefficient $\left(R^{2}=0.95\right)$. Table 3 shows a comparison of estimated values to observation data by employing a correlation function including separate fitting constants.

4.2. RRF Distribution in Heterogeneous Reservoir. To analyze the effects of heterogeneity on polymer transport, numerical 
TABLE 3: Observation and estimation of the residual resistance factor (RRF) at various permeabilities.

\begin{tabular}{lcc}
\hline $\begin{array}{l}\text { Permeability, } \\
k\left(e^{-12} \mathrm{~m}^{2}\right)\end{array}$ & $\begin{array}{c}\text { Residual resistance factor, RRF } \\
\text { (dimensionless) }\end{array}$ & $\begin{array}{c}\text { Estimated } \\
\text { values }\end{array}$ \\
\hline 2.000 & 1.9 & 1.4 \\
0.980 & 2.3 & 2.4 \\
$\mathbf{0 . 4 5 0}$ & 3.0 & 3.0 \\
0.254 & 3.9 & 4.1 \\
0.142 & 5.2 & 4.8 \\
0.069 & 7.3 & 7.4 \\
\hline
\end{tabular}

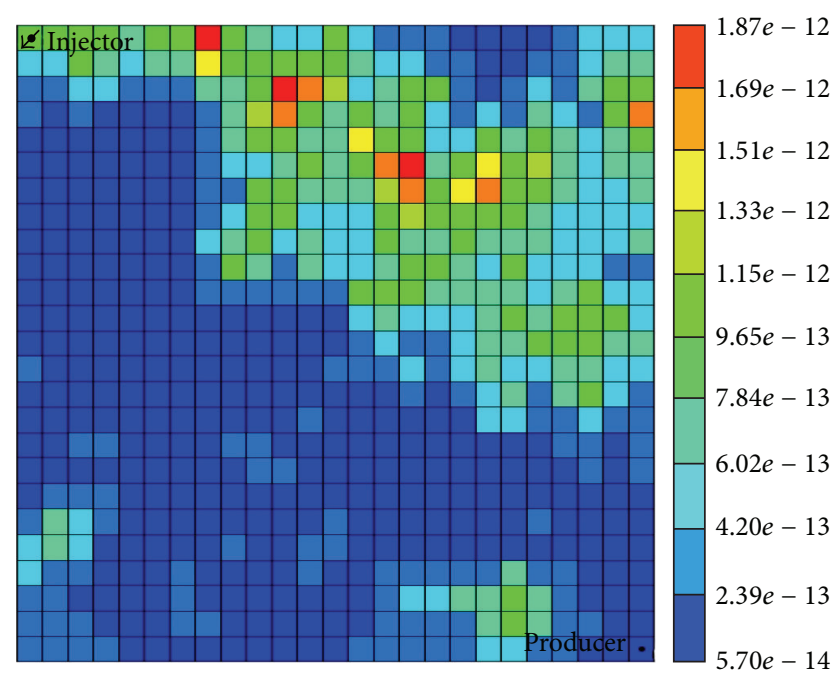

Figure 6: Permeability distribution of heterogeneous reservoir $\left(\mathrm{m}^{2}\right)$.

simulations were carried out. As shown in Figure 6, a highly heterogeneous reservoir was generated. The geometric mean of the permeability field is approximately $0.3 e^{-12} \mathrm{~m}^{2}$ in which RRF, as reference value, was set as 4.3. This reservoir was assumed to have constant porosity. Different RRF values were assigned based on four different calculations from matching models mentioned above. Polymer solution was injected into this reservoir for three years at constant injection rate of $47.67 \mathrm{~m}^{3}$ /day where total injected polymer was about three pore volumes.

Figure 7 shows distribution of RRF after three PVs of polymer injection. The first case was based on constant RRF assignment without considering the dependency of RRF on heterogeneous permeability (in other words, only dependent on concentration). Figures $7(\mathrm{~b})$ and $7(\mathrm{c})$ are the results acquired from using matching functions of RRF $=a / k^{2}+1$ and $\mathrm{RRF}=a_{1} / k^{2}+C_{\mathrm{M} \text {.Trap }}+1$. RRF resulting from employing selective mechanical trapping constant depending on permeability was displayed in Figure 7(d). The first result seems to be unrealistic even though spatial properties for polymer adsorption were significantly different. Compared with Figure 7(d), results shown in Figures 7(b) and 7(c) more likely show that there are possibilities of overestimating or underestimating prediction for the performance of polymer flooding in highly heterogeneous reservoirs. It is more accurate prediction for permeability reductions to use selective matching function allowing for the permeability dependency. Due to the heterogeneous permeability, the levels of permeability reduction are shown differently. The slightly less permeability reduction occurs in the low permeability zone for case (b) while greater reduction than real experiments data takes place in the high permeability zone for case (c). Moreover, the needs for accurate matching model of RRFdepending on permeability increase as permeability distribution is more complex and heterogeneous since in situ polymer transport can be significantly affected by RRF.

4.3. Effects of RRF on Polymer Transport. Delay of polymer propagation also affects the maintenance in polymer viscosity. The viscosity of polymer solution is a function of polymer concentration. As more polymer flows through a specific region, higher level of viscosity can be obtained. Figure 8 shows the viscosity of polymer solution calculated from different matching functions in the heterogeneous fields. There is a marked difference in the neighboring areas of the main flow, especially in relatively low permeability zones. Additionally, more preferred flow pattern is observed in case of selective fitting as shown in Figure 8(b). Compared with Figure 8(a), considerable discrepancy of permeability reduction between high and low permeability regions in Figure $8(\mathrm{~b})$ provokes nonuniform flow shape. Most of the polymer solution propagates rapidly according to high permeability region, resulting in more slanted flow tendency compared with relatively uniform flow shape shown in Figure 8(a).

In attempt to analyze relationship between polymer particle transport and permeability reduction more accurately, comparison between RRF and viscosity was made based on the proposed model. Figure 9 shows distributions of only high level of RRF $(>3.0)$ and viscosity $(>5 \mathrm{~kg} / \mathrm{m} \cdot \mathrm{s})$. The most outstanding trend is the similarity in the distributions of high level of RRF and viscosity. As expected, it is known that region with high level of RRF is roughly overlapped with highly viscous flowing region. More retained polymer particles can be accumulated in the low permeability region when the proposed model was used to calculate permeability reduction factors. The model calculates permeability reduction depending on the level of initial permeability. In other words, low permeability region becomes even lower permeability region due to the higher impairment in low permeable media than that in high permeability zone.

It is noted that the permeability reduction affects maintenance in the viscosity of polymer solution, implying RRF effects on the transported polymer concentration. The lower permeability is able to induce slower polymer propagation; thus, relatively more concentrated polymer flows through the region. Miscalculated permeability reduction can have a potential for under- or overestimation of polymer flooding. As shown in Figure 10, the observed polymer concentration at the producer shows significant differences. Use of constant RRF yields the delay in response of polymer particles compared with the proposed model. On the other hand, results 


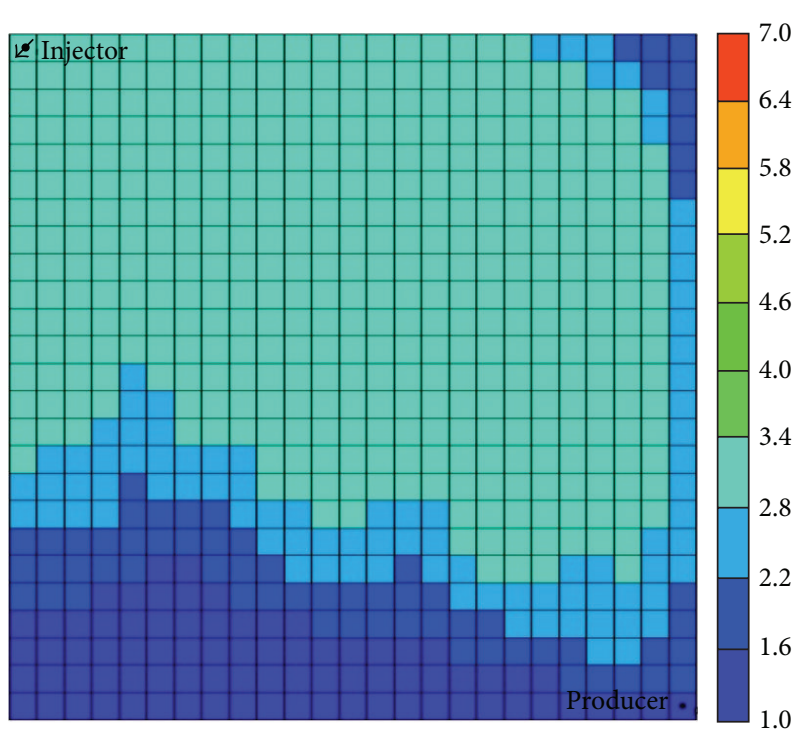

(a)

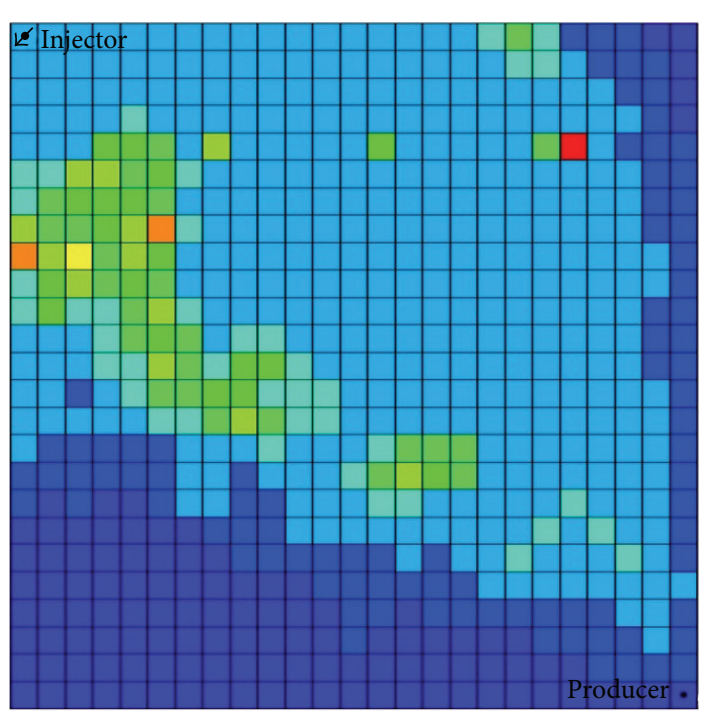

(c)

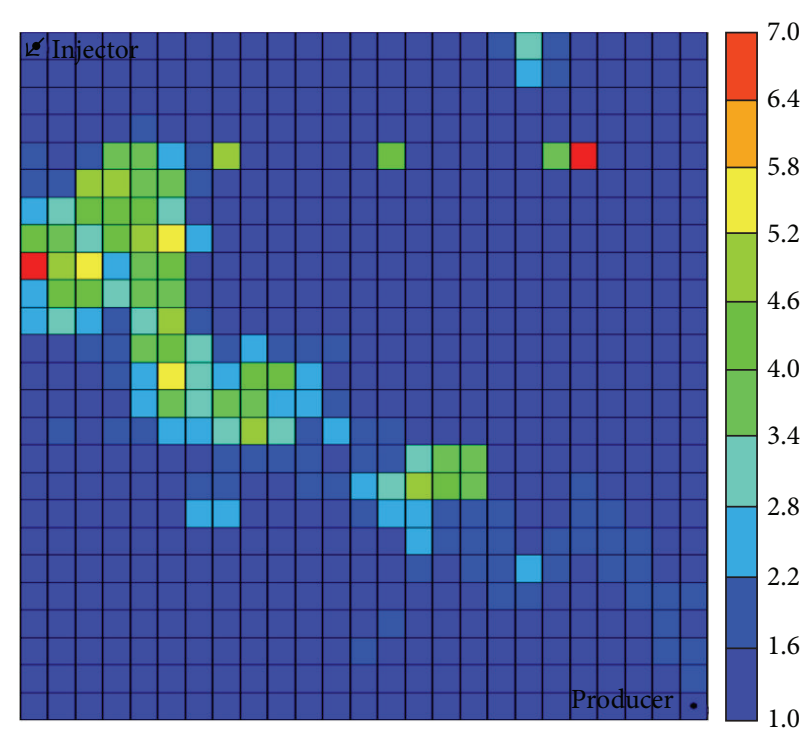

(b)

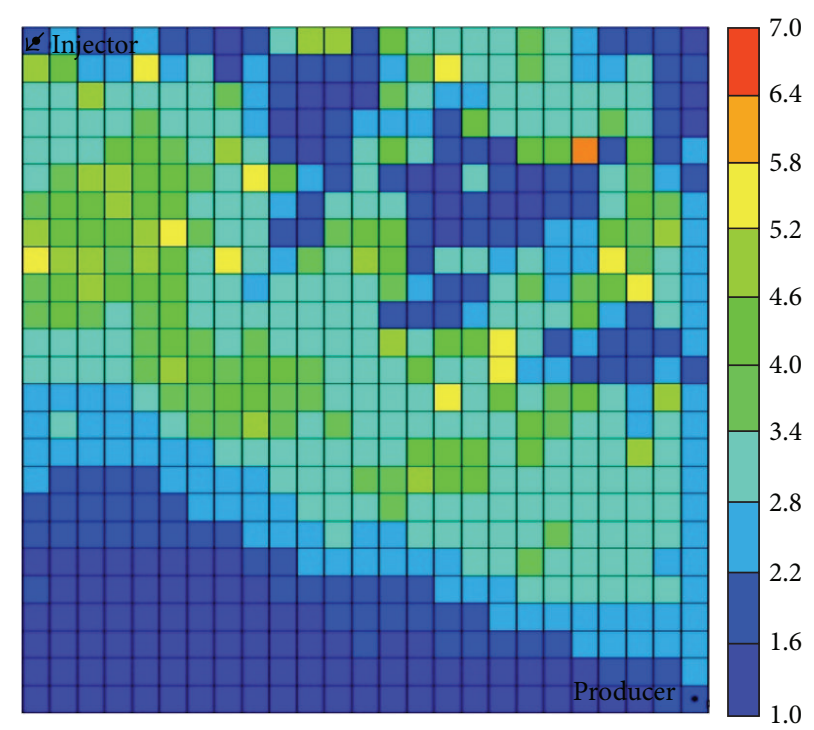

(d)

FIGURE 7: RRF distribution in heterogeneous reservoir by calculating assignment permeability reductions from four different matching functions: (a) constant RRF, (b) $\mathrm{RRF}=a / k^{2}+1$, (c) $\mathrm{RRF}=a_{1} / k^{2}+C_{\mathrm{M} \text {.Trap }}+1$, and (d) $\mathrm{RRF}=a_{1} / k^{2}+C_{\mathrm{M} \text {.Trap }}+1\left(k \leq 0.45 e^{-12} \mathrm{~m}^{2}\right)$ or $=$ $a_{2} / k^{2}+1\left(k>0.45 e^{-12} \mathrm{~m}^{2}\right)$.

from other models of (b) and (c) can be interpreted where relatively faster response of polymer in producer takes place compared with the proposed model.

\section{Conclusions}

In this study, analysis of permeability reduction factors produced from polymer retention was conducted in terms of permeability. On the basis of the results, the following conclusions were drawn:

(1) To find a matching function, applying the physical boundary condition allows matching constant $b$ to reach 1. Results from matching function correlation involving RRF reveal a low correlation coefficient and much scatter in the whole range of permeability. Using the matching results cannot verify the prediction of polymer performance in the field scale.

(2) Another fitting constant, $C_{\text {M.Trap }}$, associated with nonadsorptive permeability reduction was employed in the original form of matching function to modify the limitation that appeared in previous results. The matching performance in the low permeability zone was significantly improved, especially matching perfectly with observations for cores of 


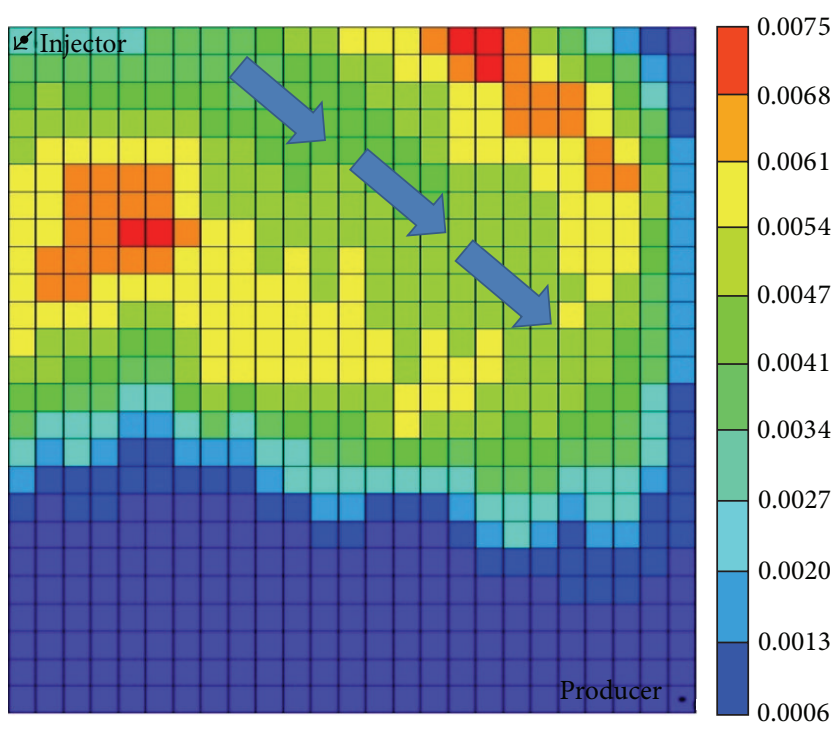

(a)

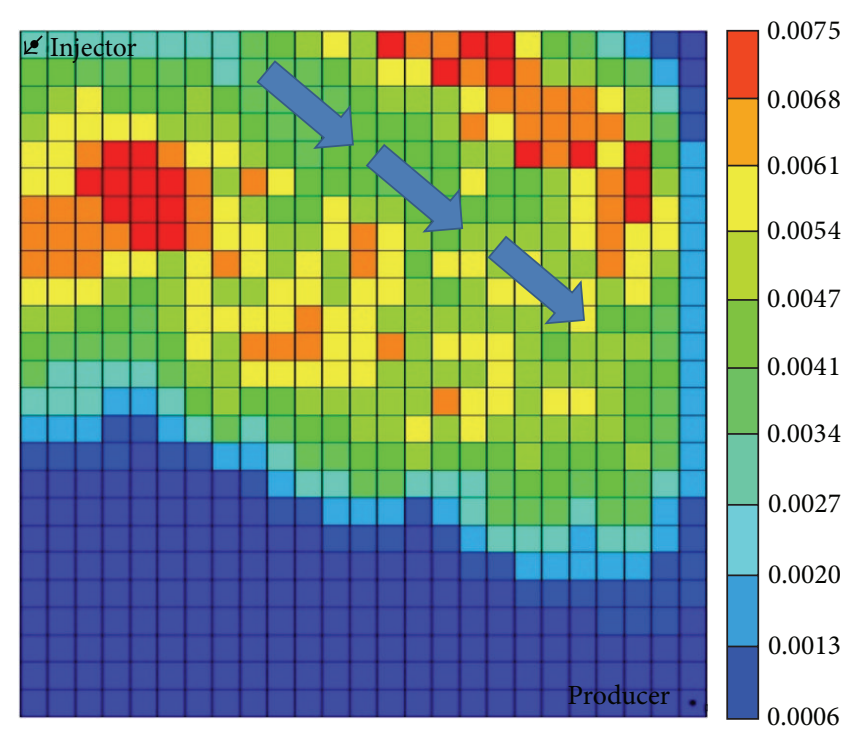

(b)

FIGURE 8: Comparison between viscosity $(\mathrm{kg} / \mathrm{m} \cdot \mathrm{s})$ distributions from using different matching functions: $(\mathrm{a})$ constant RRF and $(\mathrm{b}) \mathrm{RRF}=$ $a_{1} / k^{2}+C_{\text {M.Trap }}+1\left(k \leq 0.45 e^{-12} \mathrm{~m}^{-2}\right)$ or $=a_{2} / k^{2}+1\left(k>0.45 e^{-12} \mathrm{~m}^{-2}\right)$.

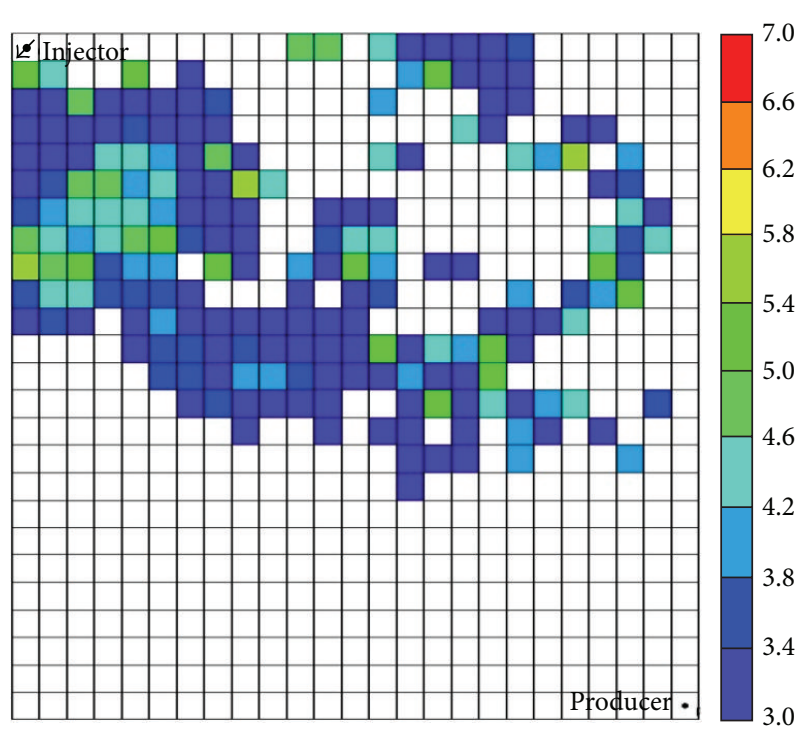

(a)

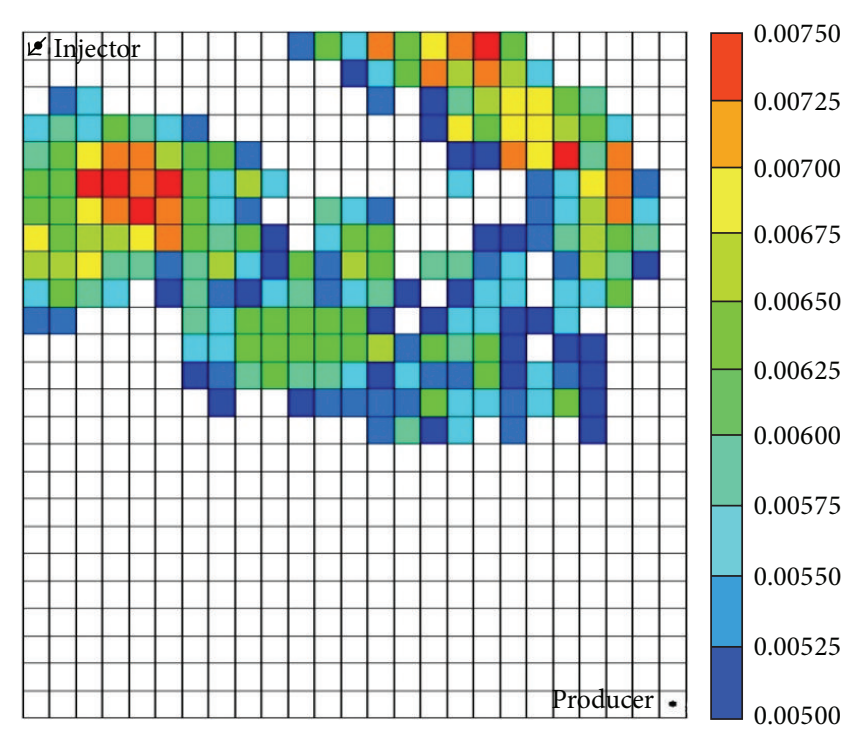

(b)

FIGURE 9: Comparison of distribution between permeability reduction and polymer viscosity regions calculated based on the proposed model: (a) RRF distribution and (b) viscosity $(\mathrm{kg} / \mathrm{m} \cdot \mathrm{s})$ distribution.

$0.45 e^{-12} \mathrm{~m}^{2}$. However, nonrealistic outputs in cases over $0.45 e^{-12} \mathrm{~m}^{2}$ were observed.

(3) To counteract the mismatching trend, the fitting constant $C_{\mathrm{M} \text { Trap }}$ was selectively applied for cores in the low permeability zone due to its physical occurrence conditions, including mechanical trapping. Results acquired from using separated functions verified good matching performance in the whole range of permeabilities. The estimated values could be implemented to predict the performance of polymer flooding under the reservoir conditions.

(4) Simulation results indicate that more accurate prediction for permeability reduction in heterogeneous reservoirs was achieved using conditional selective matching functions. Other results have possibility of overestimation or underestimation despite considering permeability dependency. Nonuniform contribution of adsorptive polymer to permeability reduction 


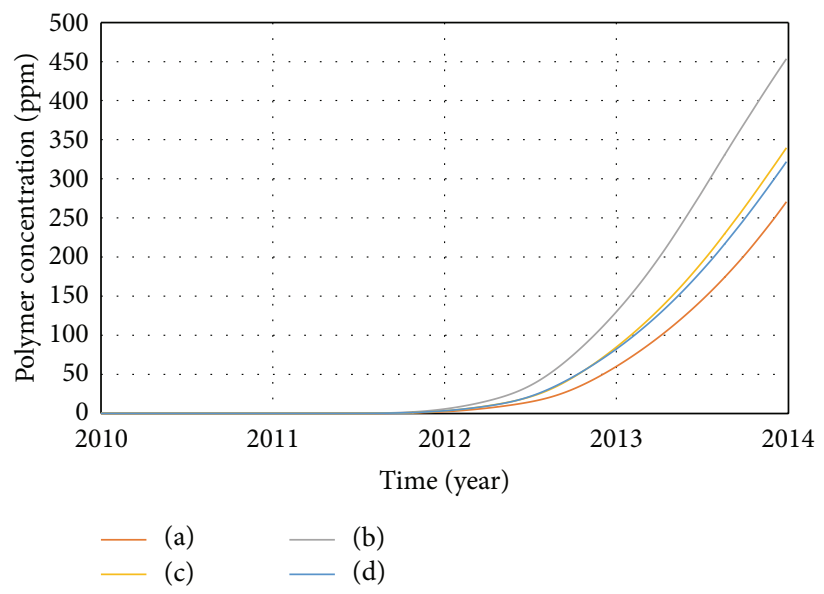

FIGURE 10: Calculated polymer concentration at producer from using four different matching functions: (a) constant RRF, (b) RRF $=a / k^{2}+1$, (c) $\mathrm{RRF}=a_{1} / k^{2}+C_{\mathrm{M} \text {.Trap }}+1$, and (d) $\mathrm{RRF}=a_{1} / k^{2}+C_{\mathrm{M} \text {.Trap }}+1\left(k \leq 0.45 e^{-12} \mathrm{~m}^{-2}\right)$ or $=a_{2} / k^{2}+1\left(k>0.45 e^{-12} \mathrm{~m}^{-2}\right)$.

depending on permeability range should be taken into account.

(5) Accurate assessment should be required in the estimation of polymer flooding performance. Permeability reduction is an important factor influencing lots of characteristics associated with polymer transport. Unless an accurate matching model is used, it is likely to show considerable difference in not only polymer viscosity, but whole flow pattern. In addition, polymer response in producer is differently calculated due to different permeability reduction factors in heterogeneous reservoirs.

We tried to find a suitable correlation function to be used in the process from lab scale to field scale. More accurate estimation can be used to predict a polymer transport in heterogeneous reservoirs, which has been previously difficult with extension process from laboratory result to field conditions. Application of selective correlation function depending on permeability condition helps to yield better results and to verify the performance of polymer flooding.

\section{Conflict of Interests}

The authors declare that there is no conflict of interests regarding the publication of this paper.

\section{Acknowledgment}

This work was supported by the Energy Efficiency \& Resources Core Technology Program of the Korea Institute of Energy Technology Evaluation and Planning (KETEP) granted financial resource from the Ministry of Trade, Industry \& Energy, Republic of Korea (no. 20122010200060).

\section{References}

[1] L. W. Lake, Enhanced Oil Recovery, Society of Petroleum Engineers, Richardson, Tex, USA, 2010.
[2] G. Chauveteau and K. Sorbie, Basic Concepts in Enhanced Oil Recovery Processes, Elsevier Science, London, UK, 1991.

[3] D. W. Green and G. P. Willhite, Enhanced Oil Recovery, Society of Petroleum Engineers, Richardson, Tex, USA, 1988.

[4] K. S. Sorbie, Polymer-Improved Oil Recovery, CRC Press, Boca Raton, Fla, USA, 2000.

[5] J. G. Dominguez and G. P. Willhite, "Retention and flow characteristics of polymer solutions in porous media," $S P E$ Journal, vol. 17, no. 2, pp. 111-121, 1977.

[6] A. D. Gupta, G. A. Pope, K. Sepehrnoori, and M. Shook, "Effects of reservoir heterogeneity on chemically enhanced oil recovery," SPE Reservoir Engineering, vol. 3, no. 2, pp. 479-488, 1988.

[7] K. Tyler, A. Henriquez, and T. Svanes, "Modeling heterogeneities in fluvial domains: a review of the influence on production profiles," in Stochastic Modeling and Geostatistics, vol. 3, chapter 8, pp. 77-89, The American Association of Petroleum Geologists, 1994.

[8] R. N. Manichand and R. Seright, "Field vs. laboratory polymerretention values for a polymer flood in the tambaredjo field," SPE Reservoir Evaluation \& Engineering, vol. 17, no. 3, pp. 314325,2014

[9] R. L. Jewett and G. F. Schurz, "Polymer flooding-a current appraisal," SPE Journal, vol. 22, no. 6, pp. 675-684, 1970.

[10] R. R. Jennings, J. H. Rogers, and T. J. West, "Factors influencing mobility control by polymer solutions," Journal of Petroleum Technology, vol. 23, no. 3, pp. 391-401, 1971.

[11] P. L. Bondor, G. J. Hirasaki, and M. J. Tham, "Mathematical simulation of polymer flooding in complex reservoirs," $S P E$ Journal, vol. 12, no. 5, pp. 317-331, 1971.

[12] C. Huh, E. A. Lange, and W. J. Cannella, "Polymer retention in porous media," in Proceedings of the SPE/DOE Enhanced Oil Recovery Symposium, Tulsa, Okla, USA, April 1990.

[13] E. J. Kolodziej, "Transport mechanisms of xanthan biopolymer solutions in porous media," in Proceedings of the SPE Annual Technical Conference and Exhibition, Houston, Tex, USA, October 1988.

[14] Y. Cohen and F. R. Christ, "Polymer retention and adsorption in the flow of polymer solutions through porous media," SPE Journal, vol. 1, no. 2, pp. 113-118, 1986.

[15] Y. Huang and K. S. Sorbie, "The adsorption and in-situ rheological behavior of Xanthan solution flowing through porous 
media," in Proceedings of the SPE/DOE Enhanced Oil Recovery Symposium, Tulsa, Okla, USA, April 1992.

[16] P. Zitha and M. Darwish, "Effect of bridging adsorption on the placement of gels for water control," in Proceedings of the SPE Asia Pacific Improved Oil Recovery Conference, Kuala Lumpur, Malaysia, October 1999.

[17] C. B. Cardoso, R. C. A. Silva, and A. P. Pires, "The role of adsorption isotherms on chemical-flooding oil recovery," in Proceedings of the SPE Annual Technical Conference and Exhibition, Anaheim, Calif, USA, November 2007.

[18] J. J. Sheng, Modern Chemical Enhanced Oil Recovery: Theory and Practice, Elservier, Oxford, UK, 2011.

[19] P. L. J. Zitha, G. Chauveteau, and A. Zaitoun, "Permeabilitydependent propagation of polyacrylamides under nearwellbore flow conditions," in SPE International Symposium on Oilfield Chemistry, San Antonio, Tex, USA, February 1995.

[20] B. Choi, K. Yu, and K. S. Lee, "Permeability-dependent retention of polymer in heterogeneous reservoirs," in Proceedings of the Offshore Technology Conference-Asia, Kuala Lumpur, Malaysia, March 2014.

[21] M. T. Szabo, "Some aspects of polymer retention in porous media using a $C^{14}$-tagged hydrolyzed polyacrylamide," SPE Journal, vol. 15, no. 4, pp. 323-337, 1975.

[22] G. J. Hirasaki and G. A. Pope, "Analysis of factors influencing mobility and adsorption in the flow of polymer solution through porous media," SPE Journal, vol. 14, no. 4, pp. 337-346, 1974.

[23] F. W. Smith, "The behavior of partially hydrolyzed polyacrylamide solutions in porous media," Journal of Petroleum Technology, vol. 22, no. 2, pp. 148-156, 1970.

[24] A. Zaitoun and N. Kohler, "Two-phase flow through porous media: effect of an adsorbed polymer layer," in Proceedings of the SPE Annual Technical Conference and Exhibition, Houston, Tex, USA, October 1988. 

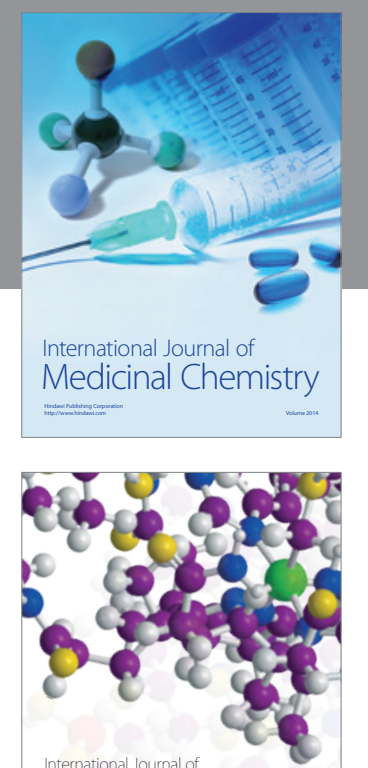

\section{Carbohydrate} Chemistry

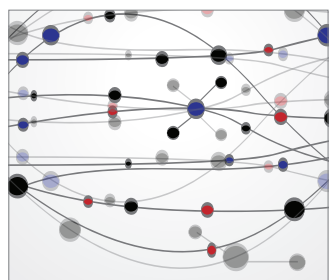

The Scientific World Journal
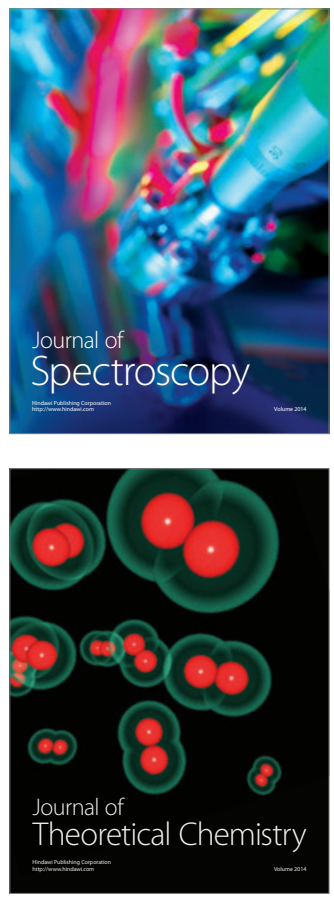
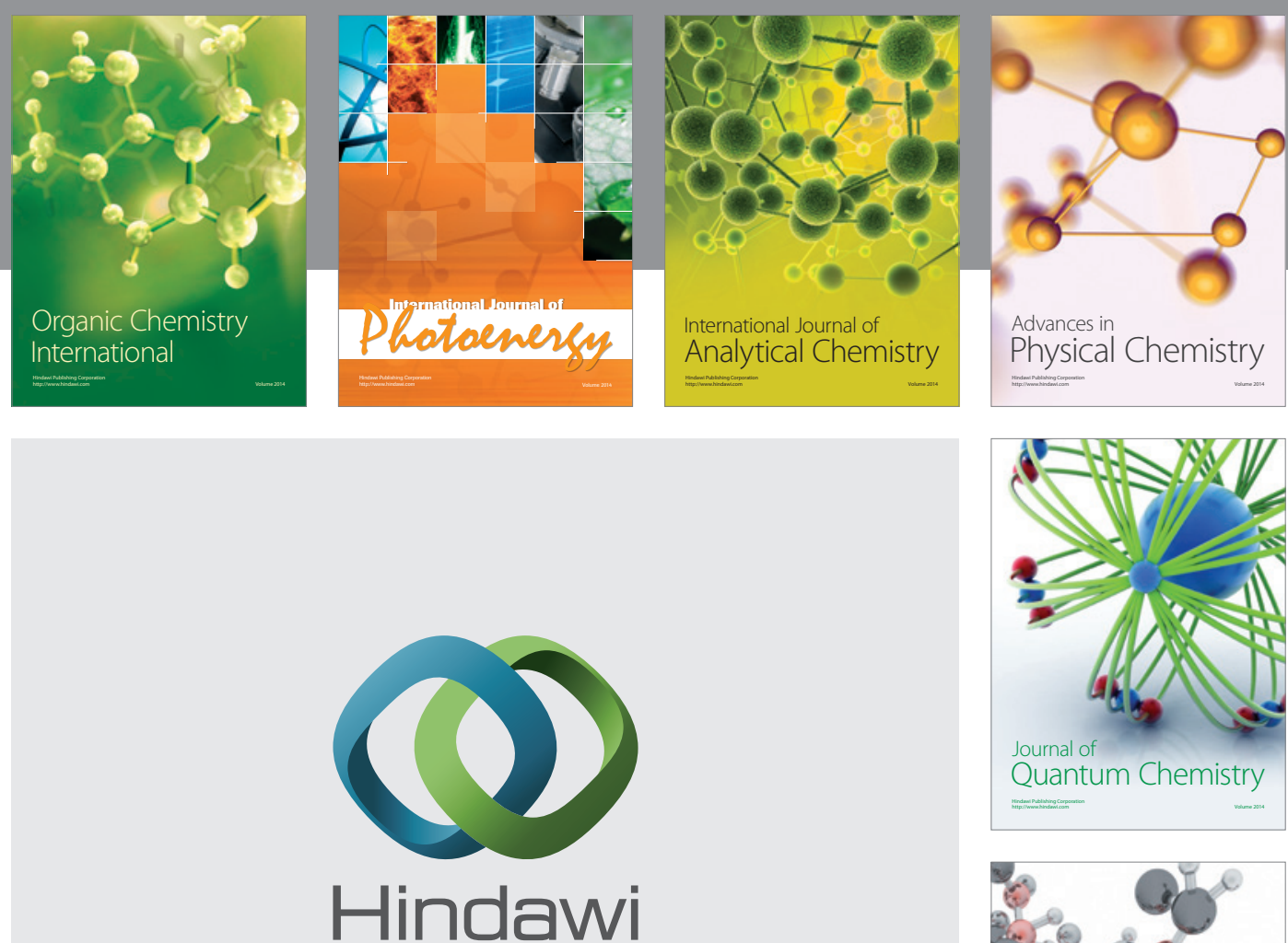

Submit your manuscripts at

http://www.hindawi.com

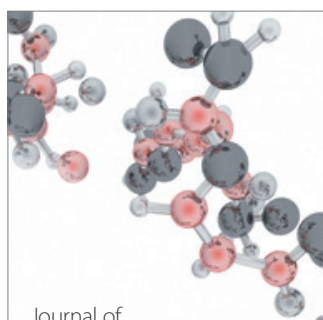

Analytical Methods

in Chemistry

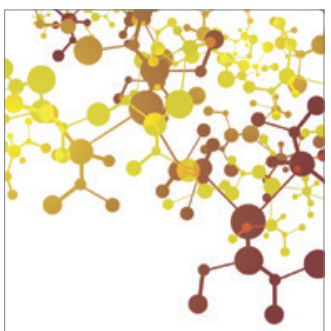

Journal of

Applied Chemistry

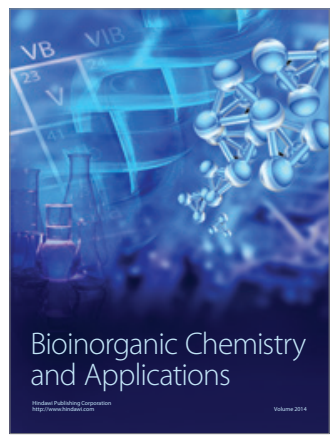

Inorganic Chemistry
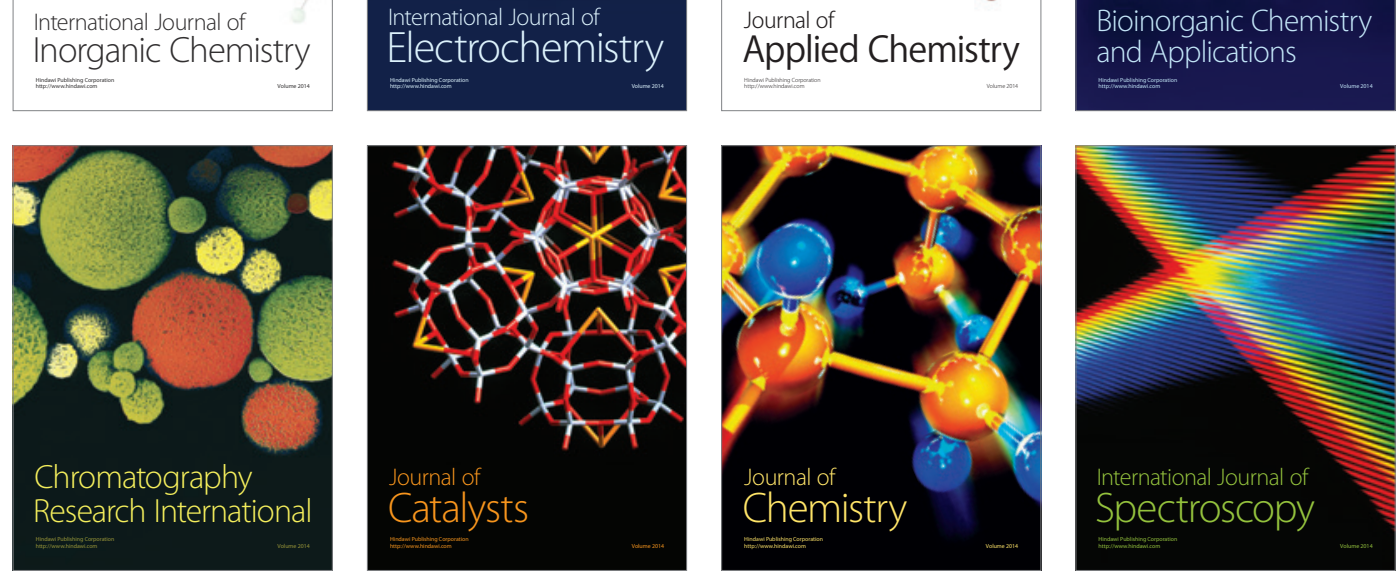